\title{
Elevation of urinary liver-type fatty acid binding protein after cardiac catheterization related to cardiovascular events
}

This article was published in the following Dove Press journal: International Journal of Nephrology and Renovascular Disease 18 August 2015

Number of times this article has been viewed

\author{
Atsuko Kamijo-Ikemori ${ }^{1,3}$ \\ Nobuyuki Hashimoto ${ }^{2}$ \\ Takeshi Sugaya' \\ Katsuomi Matsui' \\ Mikako Hisamichi' \\ Yugo Shibagaki' \\ Fumihiko Miyake ${ }^{2}$ \\ Kenjiro Kimura' \\ 'Department of Nephrology and \\ Hypertension, ${ }^{2}$ Department of \\ Cardiology, ${ }^{3}$ Department of Anatomy, \\ St Marianna University School of \\ Medicine, Kawasaki, Kanagawa, Japan
}

Correspondence: Atsuko Kamijo-lkemori Department of Anatomy, St Marianna University School of Medicine, 2-16-1 Sugao, Miyamae-Ku, Kawasaki 216-85II, Kanagawa, Japan

Tel +8I 449778 III ext 3629

Fax +8I 449777083

Email a2kamijo@marianna-u.ac.jp
Purpose: Contrast medium (CM) induces tubular hypoxia via endothelial damage due to direct cytotoxicity or viscosity. Urinary liver-type fatty acid binding protein (L-FABP) increases along with tubular hypoxia and may be a detector of systemic circulation injury. The aim of this study was to evaluate the clinical usefulness of detecting increases in urinary L-FABP levels due to administration of $\mathrm{CM}$, as a prognostic biomarker for cardiovascular disease in patients without occurrence of CM-induced nephropathy undergoing cardiac catheterization procedure (CCP). Methods: Retrospective longitudinal analyses of the relationship between urinary L-FABP levels and occurrence of cardiovascular events were performed $(n=29)$. Urinary L-FABP was measured by ELISA before CCP, and at 6, 12, 24, and 48 hours after CCP.

Results: Urinary L-FABP levels were significantly higher at 12 hours $(P<0.05)$ and 24 hours $(P<0.005)$ after CCP compared with before CCP, only in the patients with occurrence of cardiovascular events $(n=17)$, but not in those without cardiovascular events $(n=12)$. The parameter with the largest area under the curve $(0.816)$ for predicting the occurrence of cardiovascular events was the change in urinary L-FABP at 24 hours after CCP. The difference in urinary L-FABP levels ( $\triangle \mathrm{L}-\mathrm{FABP} \geq 11.0 \mu \mathrm{g} / \mathrm{g}$ creatinine) between before CCP and at 24 hours after CCP was a risk factor for the occurrence of cardiovascular events (hazard ratio, 4.93; 95\% confidence interval, $1.27-19.13 ; P=0.021)$.

Conclusion: Measurement of urinary L-FABP before CCP and at 24 hours after CCP in patients with mild to moderate renal dysfunction may be an important indicator for risk stratification of onset of cardiovascular events.

Keywords: chronic kidney disease, urinary liver-type fatty acid binding protein, L-FABP, contrast medium, urinary biomarker, cardiovascular event, renal dysfunction

\section{Introduction}

The kidneys are connected to the heart through blood vessels, and aggravating factors which lead to vascular damage, such as hyperglycemia, hypertension, hyperlipidemia, and dysregulation of the renin-angiotensin system, act on both the kidneys and heart. Thus, chronic kidney and cardiovascular diseases may coexist, which is referred to as cardiorenal syndrome. ${ }^{1}$ In patients with renal dysfunction, concurrent cardiovascular disease is directly associated with increased mortality, therefore, the development of cardiovascular events should be taken into consideration in these patients.

Urinary albumin, a biomarker of renal glomerular disease, is an independent risk factor for cardiovascular disease. ${ }^{2,3}$ As urinary albumin reflects the degree of endothelial damage in the glomerulus, it has the potential to predict future onset of cardiovascular abnormalities independent from the kidneys. ${ }^{4,5}$ Further, variation of other renal 
biomarkers that reflect the renal vascular state may also be associated with the occurrence of cardiovascular events.

Although contrast medium (CM) is indispensable for coronary angiography and X-ray imaging computed tomography, $\mathrm{CM}$ induces renal hypoperfusion and hypoxia via endothelial damage, ${ }^{6}$ due to direct cytotoxicity and viscosity of the CM. ${ }^{7}$ Therefore, patients with contrast-induced acute kidney injury (CIAKI) may have endured asymptotic endothelial damage or endothelial dysfunction that led to induction of renal hypoxia and development of CIAKI. In fact, patients with conditions that induce systemic endothelium disorder and subsequent circulation injury, such as diabetes mellitus, advanced age, hypertension and hyperlipidemia, in addition to pre-existing renal damage, are at increased risk for development of CIAKI. ${ }^{8}$ As renal and cardiac dysfunctions synergize, the presence of renal vascular injury may suggest the presence of cardiovascular injury. ${ }^{9}$ In light of these findings, we hypothesized that a biomarker which could detect renal hemodynamic change following administration of $\mathrm{CM}$, would reflect the degree of latent systemic endothelial damage and be a predictor for onset of not only CIAKI, but also future cardiovascular events.

Liver-type fatty acid binding protein (L-FABP) is expressed in the proximal tubules of the human kidney and participates in fatty acid metabolism. ${ }^{10,11}$ Urinary L-FABP was approved as a tubular injury biomarker in clinical practice by the Ministry of Health, Labour and Welfare, in Japan in $2010 .^{12}$ There is a hypoxia responsive element in the promoter region of the $L-F A B P$ gene, and other studies reported that urinary L-FABP concentration increased in parallel with decreased peritubular capillary blood flow, which was shown using noninvasive Charge Coupled Device (CCD) video recording in kidney transplant recipients, ${ }^{13}$ and this increase was associated with decreasing hemoglobin levels which led to development of renal microcirculation disorder. ${ }^{14}$ From these results, we speculated that urinary L-FABP could detect renal hemodynamic change following administration of $\mathrm{CM}$ and would be a predictor for occurrence of cardiovascular events.

Several studies indicated an increase in urinary L-FABP levels due to administration of CM in patients undergoing cardiac catheterization procedure (CCP). ${ }^{15-19}$ Although one reported that the increase in urinary L-FABP after administration of $\mathrm{CM}$ was not observed in the patients without onset of CIAKI, ${ }^{18}$ another group found that an increase in urinary L-FABP at 48 hours after administration of CM was significantly associated with decrease in renal function 1 year later in the patients undergoing CCP without occurrence of CIAKI. ${ }^{20}$ However, the correlation between increase in urinary L-FABP due to administration of CM and occurrence of cardiovascular events has not been sufficiently investigated. Therefore, we performed cross-sectional and longitudinal analyses in the current study to evaluate the clinical relevance of increase in urinary L-FABP concentration due to administration of $\mathrm{CM}$ in patients with mild to moderate renal dysfunction undergoing CCP.

\section{Patients and methods Patient selection}

In a cross-sectional study, 38 patients with mild to moderate renal dysfunction undergoing nonemergency coronary angiography or coronary intervention without occurrence of CIAKI were recruited from the outpatient clinic at the Department of Internal Medicine, St Marianna University School of Medicine Hospital (Kawasaki, Japan), between May 2005 and March 2006. The CM used in this study was nonionic, low-osmolality iodinated CM. The exclusion criteria for patients were as follows: acute coronary syndrome, acute heart failure, shock, end-stage renal disease requiring hemodialysis, infection, need of emergency coronary angiography, and intravascular administration of CM within the previous 6 days. CIAKI was defined as an absolute increase in serum creatinine of $0.5 \mathrm{mg} / \mathrm{dL}$ at 48 hours after CCP. Hydration with physiological saline at $80 \mathrm{~mL} / \mathrm{h}$ was indicated in all patients 5 hours before administration of CM and 1,000 $\mathrm{mL}$ of saline were given. This study was carried out according to the principles of the Declaration of Helsinki, and written informed consent was obtained from all of the patients. We obtained ethics approval for our study from the St. Marianna University School of Medicine Hospital, Institutional Ethics Committee.

Among the patients enrolled, those who were examined regularly at the outpatient clinic of St Marianna University School of Medicine during 2005-2014 were recruited $(n=29)$ in a retrospective longitudinal analysis. Nine patients who were excluded had changed to another hospital during the follow-up period. The primary endpoint was defined as the occurrence of cardiovascular disease (death due to a cardiovascular event, angina pectoris, nonfatal myocardial infarction, revascularization for target lesion or new lesion, nonfatal stroke, and peripheral vascular disease) and was retrospectively examined in March 2014.

\section{Study procedure}

Plasma and serum were obtained before CCP, and at 24 and 48 hours after CCP. Spot urine samples were collected before CCP, as well as 6, 12, 24, and 48 hours after CCP. 
Patients were divided into two groups based on the presence or absence of cardiovascular events during the follow-up period, and they received treatment based on the standardized strategies for diabetes mellitus, hypertension, hyperlipidemia, and cardiovascular disease during follow-up.

\section{Measurements}

\section{ELISA for measurement of urinary L-FABP}

Urinary levels of L-FABP in spot urine samples collected before CCP, and at 6, 12, 24, and 48 hours after CCP were measured by ELISA using the Human L-FABP ELISA Kit (CMIC Holdings Co, Ltd, Tokyo, Japan). ${ }^{21}$ Changes in urinary L-FABP due to administration of $\mathrm{CM}$ were analyzed using the differences in urinary L-FABP levels ( $\triangle \mathrm{L}-\mathrm{FABP})$ between before and after CCP:

$$
\begin{aligned}
\Delta \mathrm{L}-\mathrm{FABP}= & \text { value of urinary L-FABP after CCP }- \text { value } \\
& \text { of urinary L-FABP before CCP }
\end{aligned}
$$

\section{Clinical parameters}

Plasma, serum and one part of all urine samples were immediately used to measure each parameter. Serum creatinine was measured before CCP, and at 24, and 48 hours after CCP. Total cholesterol, low density lipoprotein cholesterol, serum triglyceride, high-sensitivity C-reactive protein (hs-CRP), brain natriuretic peptide, hemoglobin, and glycosylated hemoglobin were measured before undergoing CCP. In the spot urine samples, urinary creatinine, albumin, and $N$-acetyl$\beta$-D-glucosaminidase (NAG) were measured.

The levels of urinary parameters in spot urine samples were expressed as a ratio to the level of urinary creatinine. Glomerular filtration rate (GFR) was estimated using the equation proposed by the Japanese Society of Nephrology as follows: ${ }^{22}$

$$
\begin{aligned}
& \text { Estimated GFR }(\mathrm{eGFR}) ;\left(\mathrm{mL} \mathrm{min}{ }^{-1} 1.73 \mathrm{~m}^{-2}\right) \\
& =194 \times \mathrm{Cr}^{-1.094} \times \mathrm{Age}^{-0.287}(\times 0.739 \text { if female })
\end{aligned}
$$

Left ventricular ejection fraction was measured by left ventriculography, or echocardiography before, or at coronary angiography, or coronary intervention.

\section{Statistical analysis}

Values were expressed as mean \pm standard deviation. Differences in the levels of urinary parameters between before and after CCP were analyzed by the Steel-Dwass method after the Kruskal-Wallis test had been performed. To compare two groups, the Mann-Whitney $U$ test (nonparametric distribution) was used for the unpaired data.
Categorical variables were compared using the chi-square or Fisher's exact test as appropriate. Event-free rate curves of cardiovascular events were obtained using the Kaplan-Meier method and compared by log-rank test. Receiver-operating characteristic curves for urinary L-FABP levels at 12, and 24 hours after $\mathrm{CCP}$, or $\triangle \mathrm{L}$-FABP between before $\mathrm{CCP}$ and at 12 hours after CCP ( $\triangle \mathrm{L}-\mathrm{FABP}$ at 12 hours), and between before CCP and at 24 hours after CCP $(\triangle \mathrm{L}-\mathrm{FABP}$ at 24 hours) were plotted to predict the occurrence of cardiovascular events and calculate the area under the receiver-operating characteristic curves (AUC). The sensitivity, specificity, positive predictive value, and negative predictive value were calculated from $2 \times 2$ contingency tables. Cox regression analysis was performed to determine the predictor for the occurrence of cardiovascular events during the follow-up period. Unadjusted (univariate) analysis was used to select the clinical risk factor for occurrence of cardiovascular events. Following this, the unadjusted predictors with $P<0.10$ and variables with significant difference between presence and absence of cardiovascular events were entered in an adjusted (multivariate) Cox regression analysis. The hazard ratios (HRs) and 95\% confidence intervals were calculated. These statistical analyses were performed using Stat Flex 6.0 software (Artec Ltd Co, Osaka, Japan) and JMP ${ }^{\circledR} 10.0 .2$ (SAS institute Inc., Cary, NC, USA). $P$-values $<0.05$ were considered to be statistically significant.

\section{Results}

\section{Patient characteristics}

The study group at the start comprised of 38 patients (29 men) with a mean age of 65.1 years (range, 33-84 years), who had undergone coronary angiography or coronary intervention. By retrospective longitudinal analysis, we found that cardiovascular events occurred in $17(58.6 \%)$ of the 29 patients who were followed-up. The characteristics of the patients enrolled in this study are shown in Table 1 . Two patients died, ten patients developed angina pectoris or restenosis of coronary artery, two patients experienced acute myocardial infarction, one patient had a stroke, and two patients developed arteriosclerosis obliterans. With regard to age, sex, body mass index, systolic blood pressure, serum creatinine, eGFR, hs-CRP, brain natriuretic peptide, low density lipoprotein cholesterol, hemoglobin, glycosylated hemoglobin, urinary albumin, urinary NAG, urinary L-FABP, left ventricular ejection fraction (before undergoing $\mathrm{CCP}$ ), number of diseased vessels, kinds of CCP, and variety of cardiac diseases - no differences were noted between patients with and without cardiovascular events. A significantly higher percentage of 
Table I Baseline clinical characteristics of patients in the crosssection and longitudinal analyses

\begin{tabular}{|c|c|c|c|}
\hline \multirow[t]{2}{*}{ Characteristics } & \multicolumn{3}{|c|}{ Longitudinal analysis } \\
\hline & $\begin{array}{l}\text { CVD } \\
\text { group }\end{array}$ & $\begin{array}{l}\text { Non CVD } \\
\text { group }\end{array}$ & $P$-value \\
\hline $\mathrm{N}$ & 17 & 12 & \\
\hline Age (years) & $65.8 \pm 2.8$ & $69.7 \pm 3.0$ & 0.609 \\
\hline Sex (female) & $5(29.4 \%)$ & $3(25 \%)$ & 0.793 \\
\hline BMI $\left(\mathrm{kg} / \mathrm{m}^{2}\right)$ & $23.8 \pm 0.8$ & $26.3 \pm 1.7$ & 0.364 \\
\hline $\mathrm{SBP}(\mathrm{mmHg})$ & $124.1 \pm 3.9$ & $121.6 \pm 4.5$ & 0.69 \\
\hline Diabetes mellitus & $12(70.6 \%)$ & $4(33.3 \%)$ & 0.047 \\
\hline Hypertension & $15(88.2 \%)$ & $10(83.3 \%)$ & 0.706 \\
\hline Hyperlipidemia & $14(82.4 \%)$ & $4(33.3 \%)$ & 0.007 \\
\hline Baseline serum creatinine $(\mathrm{mg} / \mathrm{dL})$ & $1.3 \pm 0.1$ & $1.1 \pm 0.1$ & 0.057 \\
\hline \multicolumn{4}{|l|}{$\begin{array}{l}\text { from baseline within } 48 \mathrm{~h} \\
\text { after CCP (\%) }\end{array}$} \\
\hline eGFR $\left(\mathrm{mL} / \mathrm{min} / \mathrm{l} .73 \mathrm{~m}^{2}\right)$ & $44.4 \pm 2.7$ & $50.1 \pm 2.1$ & 0.177 \\
\hline hs-CRP (g/dL) & $0.14 \pm 0.03$ & $0.15 \pm 0.04$ & 0.815 \\
\hline BNP (pg/mL) & $156.9 \pm 60.7$ & $102.7 \pm 26.1$ & 0.786 \\
\hline LDL-C (mg/dL) & $116.3 \pm 6.8$ & $121.7 \pm 10.3$ & 0.621 \\
\hline $\mathrm{TG}(\mathrm{mg} / \mathrm{dL})$ & $185.6 \pm \mid 8.1$ & $132.7 \pm 23.4$ & 0.044 \\
\hline Hemoglobin (g/dL) & $13.2 \pm 0.6$ & $13.0 \pm 0.5$ & 0.894 \\
\hline Hemoglobin $A_{I c}(\%)$ & $5.8 \pm 0.2$ & $5.8 \pm 0.3$ & 1.000 \\
\hline Urinary albumin (mg/g creatinine) & $46.6 \pm 30.8$ & $105.0 \pm 75.5$ & 0.912 \\
\hline Urinary NAG (U/L) & $9.9 \pm 2.1$ & $9.3 \pm 2.9$ & $0.34 I$ \\
\hline Urinary FABP ( $\mu \mathrm{g} / \mathrm{g}$ creatinine) & $10.1 \pm 4.1$ & $6.2 \pm 2.0$ & 0.894 \\
\hline LVEF (\%) & $54.1 \pm 3.6$ & $54.7 \pm 4.7$ & 0.869 \\
\hline Contrast medium $(\mathrm{mL})$ & $112.9 \pm 9.6$ & $143.3 \pm 27.9$ & 1.000 \\
\hline \multicolumn{4}{|l|}{ Medication } \\
\hline RAAS inhibitor & $15(88.2 \%)$ & $6(50 \%)$ & 0.023 \\
\hline Statin & $5(29.4 \%)$ & $5(41.7 \%)$ & 0.494 \\
\hline Number of diseased vessels & $1.1 \pm 0.2$ & $0.8 \pm 0.3$ & 0.401 \\
\hline Coronary angiography & $12(70.6 \%)$ & $9(75 \%)$ & 0.793 \\
\hline Coronary intervention & $5(29.4 \%)$ & $3(25 \%)$ & 0.793 \\
\hline \multicolumn{4}{|l|}{ Type of cardiac disease } \\
\hline Ischemic heart disease & $15(88.2 \%)$ & $9(75 \%)$ & 0.289 \\
\hline Valvular disease & $0(0 \%)$ & $2(1.7 \%)$ & \\
\hline Cardiomyopathy & I (5.9\%) & I (8.3\%) & \\
\hline Pericarditis & I $(5.9 \%)$ & $0(0 \%)$ & \\
\hline
\end{tabular}

Note: Data are shown as mean \pm SD and $\mathrm{n}(\%)$.

Abbreviations: SD, standard deviation; CVD, cardiovascular disease; BMI, body mass index; SBP, systolic blood pressure; eGFR, estimated GFR; h, hour; hs-CRP, high-sensitivity CRP; BNP, brain natriuretic peptide; LDL-C, low density lipoprotein cholesterol; TG, triglycerides; LVEF, left ventricular ejection fraction; RAAS, renin-angiotensin-aldosterone system; CCP, cardiac catheterization procedure; GFR, glomerular filtration rate; CRP, C-reactive protein; NAG, $N$-acetyl- $\beta$-D-glucosaminidase; FABP, fatty acid binding protein.

patients with cardiovascular events had diabetic mellitus, and hyperlipidemia, and were taking renin-angiotensinaldosterone system (RAAS) blockers compared with those without cardiovascular events. Serum triglyceride levels were significantly higher in the former than in the latter. The dosage of CM was similar between the two groups.

\section{Changes in urinary parameters}

Significant changes in urinary albumin (Figure 1A) and urinary NAG (Figure 1B) due to administration of CM were not observed. Urinary L-FABP levels were significantly higher at 12, and 24 hours after CCP (Figure 1C). Furthermore, comparison of changes in the urinary parameter levels among patients in the two groups according to presence or absence of future cardiovascular events showed that the change in urinary albumin and urianry NAG were not found (Figure 2A and B) and that urinary L-FABP levels had increased (Figure 2C). The values of urinary L-FABP at 12, and 24 hours after CCP in the former were significantly higher than those in the latter. These results indicated that increase in urinary L-FABP due to administration of $\mathrm{CM}$ was significantly associated with the occurrence of cardiovascular events.

\section{Urinary L-FABP and occurrence of cardiovascular events}

The parameter with the largest AUC for predicting the occurrence of cardiovascular events was the $\Delta \mathrm{L}$-FABP at 24 hours (0.816), after CCP relative to pre-CCP, and the second largest AUC was urinary L-FABP level at 24 hours after CCP (0.801, Table 2). The difference between the AUCs for the two parameters was not significant $(P=0.898)$. The sensitivity and specificity of change in $\triangle \mathrm{L}-\mathrm{FABP}$ at 24 hours after $\mathrm{CCP}$, using the cut-off value of $11.0 \mu \mathrm{g} / \mathrm{g}$ creatinine, to predict cardiovascular events were 0.765 and 0.833 , respectively. Event-free rate of cardiovascular events in the patients with higher level of $\triangle \mathrm{L}-\mathrm{FABP}$ at 24 hours ( $\geq 11.0 \mu \mathrm{g} / \mathrm{g}$ creatinine) $(\mathrm{n}=16)$, was significantly lower than that in the patients with lower level of $\Delta \mathrm{L}$-FABP at 24 hours $(<11.0 \mu \mathrm{g} / \mathrm{g}$ creatinine $)$ $(\mathrm{n}=13)$ (Figure 3).

\section{Predictive factors for occurrence of cardiovascular events}

By Cox regression analysis, we found that patients with hyperlipidemia, urinary L-FABP levels at 12, and 24 hours after CCP, $\triangle \mathrm{L}-\mathrm{FABP}$ at 12 , and 24 hours after CCP, and higher level of $\triangle \mathrm{L}-\mathrm{FABP}$ at 24 hours ( $\geq 11.0 \mu \mathrm{g} / \mathrm{g}$ creatinine) were significantly associated with the occurrence of cardiovascular events (Table 3). In order to exclude the confounding factor, higher level of $\Delta \mathrm{L}-\mathrm{FABP}$ at 24 hours ( $\geq 11.0 \mu \mathrm{g} / \mathrm{g}$ creatinine) was used in the adjusted analysis because its HR in the unadjusted analysis was the highest among those of urinary L-FABP levels at 12 , and 24 hours after CCP and $\triangle \mathrm{L}-\mathrm{FABP}$ at 12 , and 24 hours after CCP. After adjustment for risk factors with $P<0.10$ found by unadjusted analysis, and for variables with significant difference between the two groups divided according to presence or absence of cardiovascular events, we found that the complication of hyperlipidemia and a higher level of $\Delta \mathrm{L}-\mathrm{FABP}$ at 24 hours ( $\geq 11.0 \mu \mathrm{g} / \mathrm{g}$ creatinine) were 

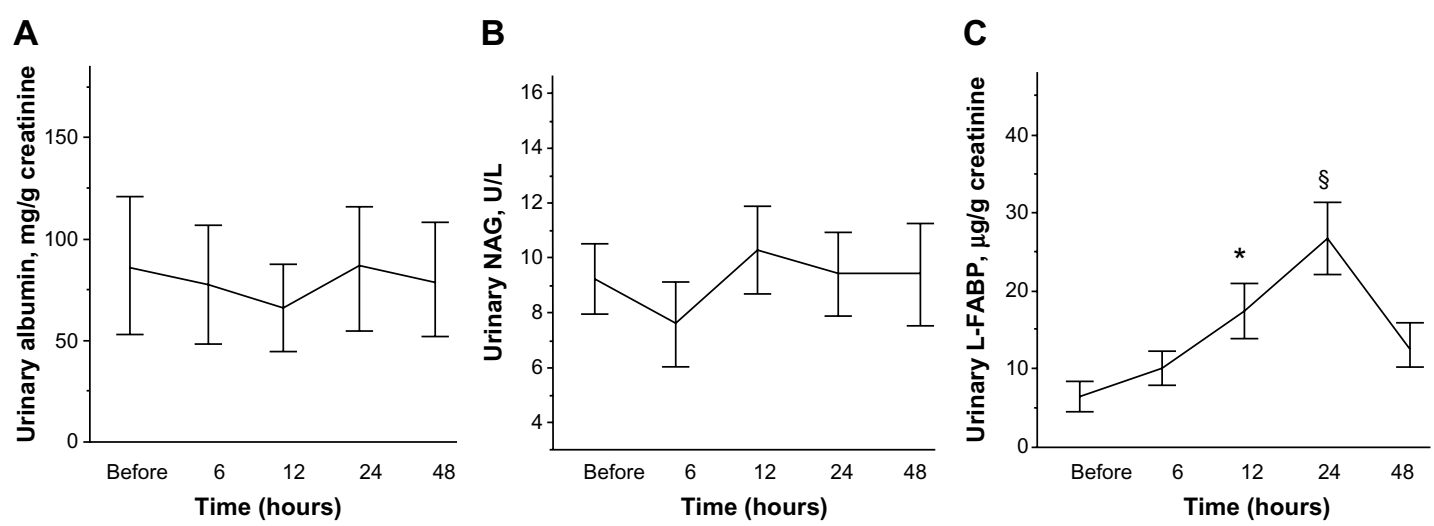

Figure I Changes in urinary albumin, NAG, and urinary L-FABP levels before and after CCP.

Notes: (A) Changes in urinary albumin levels in all patients $(n=38)$. (B) Changes in urinary NAG levels in all patients $(n=38)$. (C) Changes in urinary L-FABP levels in all the patients $(n=38)$. $* P<0.005$ compared with the value before $C C P ; \$ P<0.0001$ compared with the value before CCP.

Abbreviations: NAG, $N$-acetyl- $\beta$-D-glucosaminidase; L-FABP, liver-type fatty acid binding protein; CCP, cardiac catheterization procedure.

also associated with the occurrence of cardiovascular events (Table 3 ). When $\triangle \mathrm{L}-\mathrm{FABP}$ at 24 hours as a continuous value was used as a variable of the adjusted analysis, instead of higher level of $\triangle \mathrm{L}-\mathrm{FABP}$ at 24 hours $(\geq 11.0 \mu \mathrm{g} / \mathrm{g}$ creatinine) as a categorized variable, $\triangle \mathrm{L}-\mathrm{FABP}$ at 24 hours was demonstrated to be significantly associated with the occurrence of cardiovascular events (HR, 1.02; 95\% confidence interval, $1.00-1.05 ; P=0.044)$.

\section{Discussion}

The results of this study indicated that urinary L-FABP levels increased significantly (but not urinary albumin and urinary NAG by cross-sectional analysis) after administration of CM in patients with mild to moderate renal dysfunction undergoing CCP. In order to determine whether the increase in urinary L-FABP was associated with occurrence of cardiovascular events, a retrospective longitudinal study was performed. In patients with occurrence of cardiovascular events, urinary L-FABP levels significantly increased due to administration of CM compared to those without occurrence of cardiovascular events. In addition to hyperlipidemia, which is a classical risk factor of cardiovascular events, increase in urinary L-FABP levels at 24 hours after CCP compared with before the procedure, was found to be a risk factor for occurrence of cardiovascular events. From these results, change in urinary L-FABP after $\mathrm{CCP}$ appears to be useful for the prediction of the occurrence of cardiovascular events in patients undergoing CCP.

The mechanism by which urinary L-FABP levels increased following administration of CM was not elucidated in this study. Effects of CM on the kidney are known to induce damage of endothelial cells by direct cytotoxicity or viscosity of the CM, and consequently, can lead to
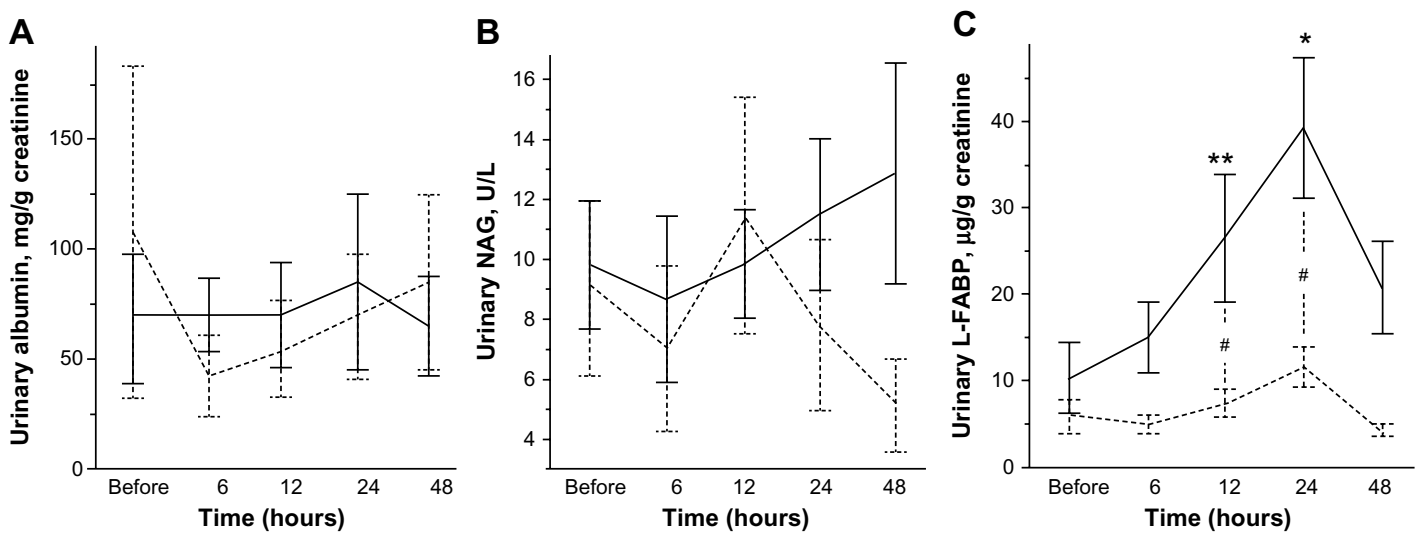

Figure 2 Changes in urinary albumin, NAG, and urinary L-FABP levels before and after CCP divided into two groups according to the occurrence of cardiovascular events. Notes: Group without occurrence of cardiovascular events (dotted line) ( $n=12$ ); group with occurrence of cardiovascular events (straight line) ( $n=17)$. (A) Changes in urinary albumin levels in the patients enrolled to the longitudinal study. (B) Changes in urinary NAG levels in the patients enrolled to the longitudinal study. (C) Changes in urinary L-FABP levels in the patients enrolled to the longitudinal study. $* P<0.005$ compared with the respective value before $C C P$; $* * P<0.05$ compared with the respective value before CCP; ${ }^{\# P}<0.05$ compared with the group without occurrence of cardiovascular events at the same time point.

Abbreviations: NAG, $N$-acetyl- $\beta$-D-glucosaminidase; L-FABP, liver-type fatty acid binding protein; CCP, cardiac catheterization procedure. 
Table 2 Usefulness of urinary L-FABP levels for predicting the occurrence of cardiovascular events

\begin{tabular}{|c|c|c|c|c|c|c|}
\hline Evaluation & AUC & $\begin{array}{l}\text { Cut-off value } \\
\text { ( } \mu \mathrm{g} / \mathrm{g} \text { creatinine) }\end{array}$ & Sensitivity & Specificity & PPV & NPV \\
\hline \multicolumn{7}{|l|}{ L-FABP } \\
\hline $12 \mathrm{~h}$ after $\mathrm{CCP}$ & 0.733 & 5.5 & 0.764 & 0.583 & 0.722 & 0.636 \\
\hline $24 \mathrm{~h}$ after $\mathrm{CCP}$ & 0.801 & 18.1 & 0.765 & 0.833 & 0.867 & 0.714 \\
\hline \multicolumn{7}{|l|}{$\Delta \mathrm{L}-\mathrm{FABP}$} \\
\hline Before-at $12 \mathrm{~h}$ & 0.74 & 2.2 & 0.706 & 0.75 & 0.8 & 0.643 \\
\hline Before-at $24 \mathrm{~h}$ & 0.816 & 11 & 0.765 & 0.833 & 0.867 & 0.714 \\
\hline
\end{tabular}

Abbreviations: AUC, area under the receiver-operating characteristic curves; PPV, positive predictive value; NPV, negative predictive value; CCP, cardiac catheterization procedure; h, hour; L-FABP, liver-type fatty acid binding protein; $\triangle \mathrm{L}-\mathrm{FABP}$, difference in urinary L-FABP.

development of renal hypoxia. ${ }^{23}$ We speculated that tubular hypoxia, induced by administration of $\mathrm{CM}$, activates hypoxia inducible factor-1 (HIF-1), ${ }^{24}$ which binds to the hypoxia responsive element in the promoter region of the L-FABP gene to up-regulate its gene expression, and promotes urinary excretion of L-FABP. In the patients with occurrence of cardiovascular events, pre-existing latent renal endothelial injury or endothelial dysfunction may be stronger, thus, marked tubular hypoxia may be induced by $\mathrm{CM}$ as compared to the patients without these events. The increase in urinary L-FABP levels after CCP may reflect the severity of pre-existing latent renal endothelial injury or endothelial dysfunction.

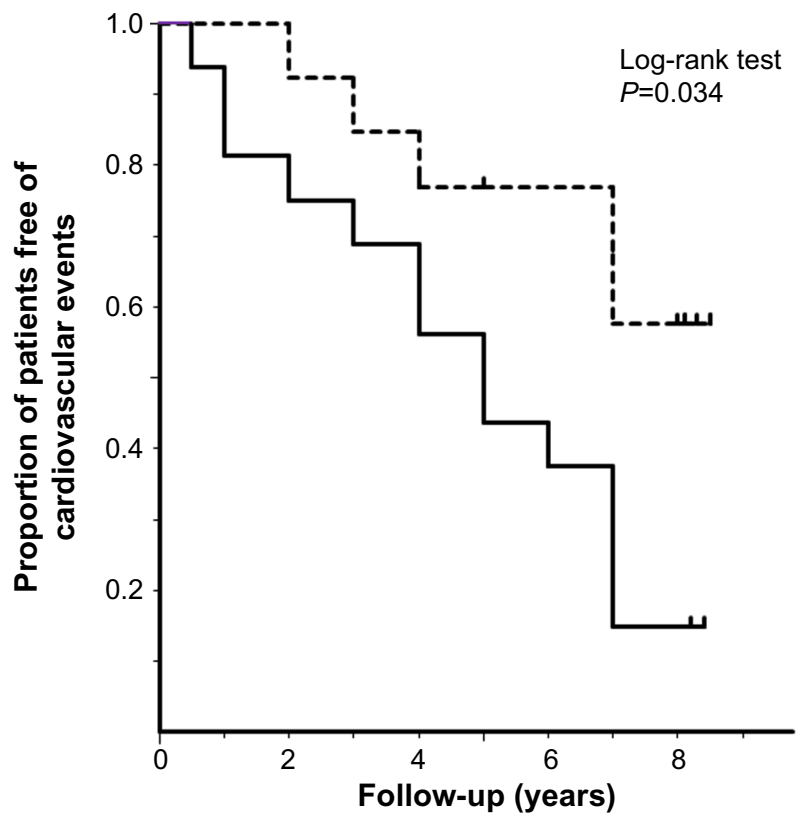

Figure 3 Event-free rate of cardiovascular events according to the Kaplan-Meier method.

Notes: Non-increase group with $\triangle \mathrm{L}-\mathrm{FABP}$ from before CCP to 24 hours after CCP $<I I .0 \mu \mathrm{g} / \mathrm{g}$ creatinine (dotted line) $(\mathrm{n}=13)$; increase group with $\triangle \mathrm{L}-\mathrm{FABP}$ before-at 24 hours $\geq 11.0 \mu \mathrm{g} / \mathrm{g}$ creatinine (straight line) $(\mathrm{n}=16)$. Differences between groups were compared by a log-rank test.

Abbreviation: $\triangle \mathrm{L}$-FABP, difference in urinary liver-type fatty acid binding protein.
Why are changes in urinary L-FABP after administration of CM associated with the occurrence of cardiovascular events? The relationship between urinary L-FABP and cardiovascular disease has been examined in some studies. In a previous prospective study performed in type 2 diabetic patients with normoalbuminuria and microalbuminuria, the occurrence of cardiovascular events in addition to the progression to end-stage renal failure were observed at a greater rate in patients with higher urinary L-FABP. ${ }^{25}$ Moreover, a higher urinary L-FABP level was a predictor for all-cause mortality, independent of urinary albumin and other established risk factors in type 1 diabetic patients with normoalbuminuria. ${ }^{26}$ A recent cross-sectional investigation that enrolled type 2 diabetic patients with normoalbuminuria showed that the frequency of electrocardiographic abnormalities in patients with a higher level of urinary L-FABP (greater than upper limit of reference value of urinary L-FABP, $>8.4 \mu \mathrm{g} / \mathrm{g}$ creatinine), ${ }^{27}$ was significantly higher than those with a lower level of urinary L-FABP $(<8.4 \mu \mathrm{g} / \mathrm{g}$ creatinine), although urinary albumin and eGFR levels using standardized serum cystatin C were similar between the two groups. ${ }^{28}$ Urinary L-FABP may reflect the degree of not only renal microcirculation injury, but also systemic microcirculation injury, and may be a predictive marker for the onset of cardiovascular events. Furthermore, another group also reported that increase in urinary L-FABP at 24 hours after CCP, but not before undergoing $\mathrm{CCP}$, was significantly associated with the onset of cardiovascular disease in the patients who were followed for up to 4.5 years, although cut-off values of the degree of increase in urinary L-FABP and changes in urinary L-FABP before and after CCP were not shown. ${ }^{17}$ From these results, urinary L-FABP may sensitively detect renal hemodynamic change following administration of $\mathrm{CM}$, which induces renal hypoxia, and the change in urinary L-FABP by CM may depend on the degree of underlying asymptomatic renal circulation disorder. As renal circulation disorder is relevant to systemic circulation injury, response of urinary L-FABP 
Table 3 Univariate and multivariate Cox regression analyses using the occurrence of cardiovascular events

\begin{tabular}{|c|c|c|c|c|c|c|}
\hline \multirow[t]{2}{*}{ Variables } & \multicolumn{3}{|c|}{ Univariate analysis } & \multicolumn{3}{|c|}{ Multivariate analysis } \\
\hline & Hazard ratio & $95 \% \mathrm{Cl}$ & $P$-value & Hazard ratio & $95 \% \mathrm{Cl}$ & $P$-value \\
\hline Age, years & 0.98 & $0.94-1.03$ & 0.427 & & & \\
\hline Sex (male) & 0.75 & $0.26-2.14$ & 0.593 & & & \\
\hline Smoking & 1.24 & $0.79-1.94$ & 0.353 & & & \\
\hline \multicolumn{7}{|l|}{ Previous history } \\
\hline Diabetes mellitus & 2.40 & $0.84-6.82$ & 0.101 & 2.28 & $0.73-7.12$ & 0.157 \\
\hline Hypertension & 1.16 & $0.27-5.10$ & 0.840 & & & \\
\hline Hyperlipidemia & 4.39 & $1.24-15.49$ & 0.021 & 5.09 & $1.30-19.86$ & 0.019 \\
\hline $\mathrm{BMI}, \mathrm{kg} / \mathrm{m}^{2}$ & 0.91 & $0.79-1.04$ & 0.177 & & & \\
\hline $\mathrm{SBP}, \mathrm{mmHg}$ & 1.01 & $0.98-1.04$ & 0.50 & & & \\
\hline LVEF, \% & 1.00 & $0.97-1.04$ & 0.854 & & & \\
\hline Hemoglobin $\mathrm{A}_{1 \mathrm{c}}, \%$ & 1.08 & $0.61-1.90$ & 0.801 & & & \\
\hline eGFR, $\mathrm{mL} / \mathrm{min} / 1.73 \mathrm{~m}^{2}$ & 0.97 & $0.93-1.01$ & 0.199 & & & \\
\hline hs-CRP & 1.09 & $0.07-18.25$ & 0.950 & & & \\
\hline BNP & 1.00 & $0.99-1.00$ & 0.750 & & & \\
\hline LDL, mg/dL & 0.99 & $0.98-1.02$ & 0.99 & & & \\
\hline $\mathrm{TG}, \mathrm{mg} / \mathrm{dL}$ & 1.00 & $0.99-1.01$ & 0.164 & 1.01 & $0.99-1.02$ & 0.064 \\
\hline \multicolumn{7}{|l|}{ Urinary albumin, $\mathrm{mg} / \mathrm{g}$ creatinine } \\
\hline Before CCP & 0.99 & $0.99-1.00$ & 0.621 & & & \\
\hline \multicolumn{7}{|l|}{ Urinary L-FABP, $\mu g / g$ creatinine } \\
\hline Before CCP & 1.02 & $0.98-1.05$ & $0.36 \mathrm{I}$ & & & \\
\hline $12 \mathrm{~h}$ after $\mathrm{CCP}$ & 1.02 & $1.00-1.03$ & 0.028 & & & \\
\hline $24 \mathrm{~h}$ after $\mathrm{CCP}$ & 1.02 & $1.01-1.03$ & 0.007 & & & \\
\hline \multicolumn{7}{|l|}{$\Delta \mathrm{L}-\mathrm{FABP}, \mu \mathrm{g} / \mathrm{g}$ creatinine } \\
\hline Before-at $12 \mathrm{~h}$ & 1.03 & $1.01-1.05$ & 0.015 & & & \\
\hline Before-at $24 \mathrm{~h}$ & 1.03 & $1.01-1.05$ & 0.003 & & & \\
\hline Higher level of $\triangle \mathrm{L}-\mathrm{FABP}$ at & 3.60 & I.I7-II.I & 0.026 & 4.93 & $1.27-19.13$ & 0.021 \\
\hline \multicolumn{7}{|l|}{$24 \mathrm{~h} \geq \mathrm{I} I .0 \mu \mathrm{g} / \mathrm{g}$ creatinine } \\
\hline RAAS inhibitor & 3.58 & $0.82-15.68$ & 0.091 & 0.65 & $0.11-3.97$ & 0.642 \\
\hline $\begin{array}{l}\text { Presence of ischemic heart } \\
\text { disease at the start }\end{array}$ & 1.86 & $0.65-5.33$ & 0.245 & & & \\
\hline
\end{tabular}

Note: Bold text indicates signficant results.

Abbreviations: BMI, body mass index; SBP, systolic blood pressure; LVEF, left ventricular ejection fraction; eGFR, estimated GFR; hs-CRP, high-sensitivity CRP; BNP, brain natriuretic peptide; LDL, low density lipoprotein; TG, triglycerides; CCP, cardiac catheterization procedure; RAAS, renin-angiotensin-aldosterone system; Cl, confidence interval; h, hour; GFR, glomerular filtration rate; CRP, C-reactive protein; L-FABP, liver-type fatty acid binding protein; $\triangle \mathrm{L}-\mathrm{FABP}$, difference in urinary L-FABP.

following $\mathrm{CM}$ administration may be a predictor for onset of future cardiovascular events.

The present study and a previous study, ${ }^{17}$ indicated that measurement of urinary L-FABP before CCP and at 24 hours after CCP was important for risk stratification of the onset of future cardiovascular events in the patients undergoing CCP with mild to moderate renal dysfunction. In a previous clinical study of CIAKI in patients undergoing CCP, a higher level of urinary L-FABP before CIAKI was significantly correlated with the occurrence of CIAKI, ${ }^{16,18}$ and the cut-off value of urinary L-FABP, $24.5 \mathrm{mg} / \mathrm{g}$ creatinine, was found. ${ }^{16}$ Furthermore, increase in urinary L-FABP at 48 hours after CCP was relevant to short-term renal dysfunction in patients undergoing $\mathrm{CCP}$ without occurrence of CIAKI. ${ }^{20}$ From these studies, measurement of urinary L-FABP before undergoing CCP, and at 24, and 48 hours after CCP may be useful for prediction of the occurrence of CIAKI, short-term renal prognosis and onset of future cardiovascular events.

Urinary albumin is a predictive, independent risk factor for cardiovascular events. ${ }^{29,30}$ However, urinary albumin level before undergoing CCP in this study was not correlated with the occurrence of cardiovascular events. In the cohort of this study, the mean urinary albumin level was low, and there were only four patients with microalbuminuria and two patients with macroalbuminuria. Occurrence of cardiovascular events was observed even in the patients with normoalbuminuria. With regard to urinary NAG as a tubular damage marker, change in urinary NAG was not observed due to administration of $\mathrm{CM}$. This result indicated that tubular structural damage was not induced by the CM used in this study. Hs-CRP is a marker for systemic inflammation, which leads to arteriosclerosis, and increase in hs-CRP level is a risk factor for occurrence of cardiovascular disease..$^{31,32}$ 
Hs-CRP levels in this study were relatively low and similar between the patients with the presence, or absence of occurrence of cardiovascular events. In the patients with cardiac disease showing low levels of hs-CRP, occurrence of cardiovascular events may be weakly associated with the levels of hs-CRP.

One of the limitations of this study was that the number of subjects was small, and a prospective large-scale multicenter study is needed to reconfirm the relationship between increase in urinary L-FABP due to administration of CM and occurrence of cardiovascular events, and the cut-off values for identifying high-risk patients who develop cardiovascular events. Secondly, the present study was designed as a retrospective observational study, and treatments depended on the discretion of the individual cardiologists. The number of patients taking RAAS inhibitors was larger in the group with occurrence of cardiovascular disease than in the group without it. We considered that if activation of RAAS was not sufficiently controlled, this may have led to the occurrence of cardiovascular events. Finally, the type of cardiac disease was not uniform in the patients enrolled to this study. However, the type of cardiac disease was not significantly different between the patients with the presence, or absence of occurrence of cardiovascular events.

In summary, the current study found that measurement of urinary L-FABP before CCP and at 24 hours after CCP, and the evaluation of the difference between them, may be a suitable biomarker to predict the onset of cardiovascular events in clinical practice in patients with mild to moderate renal dysfunction undergoing CCP.

\section{Acknowledgments}

We are grateful to Ms Seiko Hoshino, Kimie Katayama, Aya Sakamaki, and Sanae Ogawa for assistance with the collection of urine and serum samples. We would also like to thank Dr Hiroyo Sasaki for obtaining informed consent from the patients.

\section{Disclosure}

T Sugaya is the Director and Senior Scientist of CMIC Holdings Co, Ltd, the company that produced the kits for L-FABP analysis. None of the other authors have conflicts of interest or financial disclosures of any relevance to the present study.

\section{References}

1. Shah BN, Greaves K. The Cardiorenal Syndrome: A review. Int J Nephrol. 2010;2011:920195.
2. Sarnak MJ, Levey AS, Schoolwerth AC, et al. Kidney disease as a risk factor for development of cardiovascular disease: A statement from the American Heart Association Councils on Kidney in Cardiovascular Disease, High Blood Pressure Research, Clinical Cardiology, and Epidemiology and Prevention. Circulation. 2003;108(17):2154-2169.

3. Diercks GF, van Boven AJ, Hillege HL, et al. Microalbuminuria is independently associated with ischaemic electrocardiographic abnormalities in a large non-diabetic population. The PREVEND (Prevention of REnal and Vascular ENdstage Disease) study. Eur Heart $J$. 2000;21(23):1922-1927.

4. Deckert T, Feldt-Rasmussen B, Borch-Johnsen K, Jensen T, KofoedEnevoldsen A. Albuminuria reflects widespread vascular damage. The Steno hypothesis. Diabetologia. 1989;32(4):219-226.

5. Stehouwer CD, Henry RM, Dekker JM, Nijpels G, Heine RJ, Bouter LM. Microalbuminuria is associated with impaired brachial artery, flow-mediated vasodilation in elderly individuals without and with diabetes: Further evidence for a link between microalbuminuria and endothelial dysfunction - the Hoorn Study. Kidney Int Suppl. 2004;(92):S42-S44.

6. Sendeski MM, Persson AB, Liu ZZ, et al. Iodinated contrast media cause endothelial damage leading to vasoconstriction of human and rat vasa recta. Am J Physiol Renal Physiol. 2012;303(12):F1592-F1598.

7. Seeliger E, Sendeski M, Rihal CS, Persson PB. Contrast-induced kidney injury: Mechanisms, risk factors, and prevention. Eur Heart $J$. 2012;33(16):2007-2015.

8. Franke RP, Jung F. Pathophysiology of the contrast media-induced nephropathy (CIN) in patients undergoing coronary interventions. Clin Hemorheol Microcirc. 2013;53(1-2):143-153.

9. Ronco C, Haapio M, House AA, Anavekar N, Bellomo R. Cardiorenal syndrome. J Am Coll Cardiol. 2008;52(19):1527-1539.

10. Veerkamp JH, Peeters RA, Maatman RG. Structural and functional features of different types of cytoplasmic fatty acid-binding proteins. Biochim Biophys Acta. 1991;1081(1):1-24.

11. Veerkamp JH, van Kuppevelt TH, Maatman RG, Prinsen CF. Structural and functional aspects of cytosolic fatty acid-binding proteins. Prostaglandins Leukot Essent Fatty Acids. 1993;49(6):887-906.

12. Kamijo-Ikemori A, Sugaya T, Ichikawa D, et al. Urinary liver type fatty acid binding protein in diabetic nephropathy. Clin Chim Acta. 2013;424:104-108.

13. Yamamoto T, Noiri E, Ono Y, et al. Renal L-type fatty acid - binding protein in acute ischemic injury. J Am Soc Nephrol. 2007;18(11): 2894-2902.

14. Eynatten von M, Baumann M, Heemann U, et al. Urinary L-FABP and anaemia: distinct roles of urinary markers in type 2 diabetes. Eur J Clin Invest. 2010;40:95-102.

15. Fukuda Y, Miura S, Zhang B, et al. Significance of urinary liver-fatty acid-binding protein in cardiac catheterization in patients with coronary artery disease. Intern Med. 2009;48(19):1731-1737.

16. Manabe K, Kamihata H, Motohiro M, Senoo T, Yoshida S, Iwasaka T. Urinary liver-type fatty acid-binding protein level as a predictive biomarker of contrast-induced acute kidney injury. Eur J Clin Invest. 2012; 42(5):557-563.

17. Matsumori R, Shimada K, Kiyanagi T, et al. Clinical significance of the measurements of urinary liver-type fatty acid binding protein levels in patients with acute coronary syndrome. J Cardiol. 2012;60(3): 168-173.

18. Nakamura T, Sugaya T, Node K, Ueda Y, Koide H. Urinary excretion of liver-type fatty acid-binding protein in contrast medium-induced nephropathy. Am J Kidney Dis. 2006;47(3):439-444.

19. Kato K, Sato N, Yamamoto T, Iwasaki YK, Tanaka K, Mizuno K. Valuable markers for contrast-induced nephropathy in patients undergoing cardiac catheterization. Circ J. 2008;72(9):1499-1505.

20. Fujita D, Takahashi M, Doi K, et al. Response of urinary liver-type fatty acid-binding protein to contrast media administration has a potential to predict one-year renal outcome in patients with ischemic heart disease. Heart Vessels. 2015;30(3):296-303. 
21. Kamijo A, Kimura K, Sugaya T, et al. Urinary fatty acid binding protein as a new clinical marker for the progression of chronic renal disease. $J$ Lab Clin Med. 2004;143:23-30.

22. Matsuo S, Imai E, Horio M, et al. Revised equations for estimated GFR from serum creatinine in japan. Am J Kidney Dis. 2009;53(6): 982-992.

23. Wong PC, Li Z, Guo J, Zhang A. Pathophysiology of contrast-induced nephropathy. Int J Cardiol. 2012;158(2):186-192.

24. Nangaku M. Chronic hypoxia and tubulointerstitial injury: A final common pathway to end-stage renal failure. J Am Soc Nephrol. 2006; 17(1):17-25

25. Araki S, Haneda M, Koya D, et al. Predictive effects of urinary liver-type fatty acid-binding protein for deteriorating renal function and incidence of cardiovascular disease in type 2 diabetic patients without advanced nephropathy. Diabetes Care. 2013;36(5):1248-1253.

26. Nielsen SE, Sugaya T, Hovind P, Baba T, Parving HH, Rossing P. Urinary liver-type fatty acid-binding protein predicts progression to nephropathy in type 1 diabetic patients. Diabetes Care. 2010;33(6): $1320-1324$.
27. Kamijo-Ikemori A, Sugaya T, Yasuda T, et al. Clinical significance of urinary liver-type fatty acid-binding protein in diabetic nephropathy of type 2 diabetic patients. Diabetes Care. 2011;34(3):691-696.

28. Maeda Y, Suzuki A, Ishii J, et al. Level of urinary liver-type fatty acidbinding protein is associated with cardiac markers and electrocardiographic abnormalities in type- 2 diabetes with chronic kidney disease stage g1 and g2. Heart Vessels. 2015;30(3):362-368.

29. Weir MR. Microalbuminuria and cardiovascular disease. Clin J Am Soc Nephrol. 2007;2(3):581-590.

30. Stehouwer CD, Smulders YM. Microalbuminuria and risk for cardiovascular disease: Analysis of potential mechanisms. J Am Soc Nephrol. 2006;17(8):2106-2111.

31. Kaptoge S, Di Angelantonio E, Lowe G, et al. C-reactive protein concentration and risk of coronary heart disease, stroke, and mortality: an individual participant meta-analysis. Lancet. 2010;375(9709): $132-140$.

32. Ridker PM. Clinical application of C-reactive protein for cardiovascular disease detection and prevention. Circulation. 2003;107(3):363-369.

\section{Publish your work in this journal}

The International Journal of Nephrology and Renovascular Disease is an international, peer-reviewed open-access journal focusing on the pathophysiology of the kidney and vascular supply. Epidemiology, screening, diagnosis, and treatment interventions are covered as well as basic science, biochemical and immunological studies. The journal welcomes original research, clinical studies, reviews \& evaluations, expert opinion and commentary, case reports and extended reports. The manuscript management system is completely online and includes a very quick and fair peerreview system, which is all easy to use. Visit http://www.dovepress.com/ testimonials.php to read real quotes from published authors

Submit your manuscript here: http://www.dovepress.com/international-journal-of-nephrology-and-renovascular-disease-journal 